Conclusion RR identified significantly fewer included studies than SR, but there were no significant differences in the review results. RR offered greater efficiency with far greater $\%$ of reviewed records being included. This analysis benefited from using data from a large scale epidemiological review. However, it only assessed one broad topic area. Further research and evidence synthesis is needed to assess the value of RR in an epidemiological setting.

\section{Mental Health 2}

\section{OP83 HOW MUCH OF THE EFFECT OF DISABILITY ACQUISITION ON MENTAL HEALTH IS MEDIATED THROUGH EMPLOYMENT AND INCOME? A CAUSAL MEDIATION ANALYSIS QUANTIFYING INTERVENTIONAL INDIRECT EFFECTS USING LONGITUDINAL DATA FROM WORKING AGE AUSTRALIANS}

${ }^{1} \mathrm{Z}$ Aitken*, ${ }^{1} \mathrm{JA}$ Simpson, ${ }^{1} \mathrm{R}$ Bentley, ${ }^{1,2} \mathrm{~T}$ Blakely, ${ }^{1} \mathrm{AM}$ Kavanagh. ${ }^{1}$ Melbourne School of Population and Global Health, The University of Melbourne, Melbourne, Australia; ${ }^{2}$ Department of Public Health, The University of Otago, Wellington, New Zealand

10.1136/jech-2019-SSMabstracts.86

Background There is evidence that disability acquisition causes a decline in mental health, but few studies have examined the causal mechanisms through which the effect operates. Our previous research examined three broad groups of socioeconomic characteristics and found that material factors, rather than psychosocial or behavioural, predominately mediated the effect. However, there remains a lack of understanding about which specific material factors drive the association, limiting the development of effective interventions to improve the mental health of people with disabilities. This study examined how much changes to employment and income mediate the effect of disability acquisition on mental health.

Methods We used data from the Household, Income and Labour Dynamics in Australia Survey, a longitudinal study of Australian households, which collects information annually on a wide range of demographic, social, economic, and health characteristics. We used four waves of data (2011-2014) to compare self-reported mental health between working aged individuals who acquired a disability $(n=233)$ and those who remained disability-free $(n=5419)$. Mental health was measured using the mental health inventory, a subscale of the SF36 health questionnaire. We conducted a causal mediation analysis to quantify interventional indirect effects of disability acquisition on mental health operating through two distinct mediators: employment status and household income. This novel method enables quantification of path-specific effects operating through multiple mediators. We used multiple imputation with 50 imputed datasets to account for missing data and conducted analyses in Stata/SE 15.

Results Disability acquisition was estimated to cause a fivepoint decline in mental health (estimated mean difference: $-4.8,95 \%$ CI $-7.0,-2.7)$. The interventional indirect effect through employment was estimated to be a 0.5 point difference $(-0.5,95 \%$ CI $-1.0,0.0)$, accounting for $11 \%$ of the total effect, whereas there was no evidence that income explained any of the effect.

Conclusion Using a novel approach to mediation analysis which addresses limitations of traditional mediation methods and can be estimated under weaker assumptions than natural indirect effects, we found that some of the effect of disability acquisition on mental health was explained by changes to employment, but not income. Key limitations included strong assumptions about unmeasured confounding and the small number of people acquiring a disability in our dataset. The findings have important policy implications, highlighting the need to improve employment opportunities and vocational training for people who acquire a disability to improve mental health and reduce current mental health inequalities experienced by people with disabilities.

\section{OP84 COULD INCREASING INCOME IN LONE-PARENT HOUSEHOLDS REDUCE POPULATION PREVALENCE AND INEQUALITIES IN CHILDREN'S MENTAL HEALTH PROBLEMS? A POLICY SIMULATION IN THE UK MILLENNIUM COHORT STUDY}

${ }^{1} S$ Hope ${ }^{*},{ }^{2}$ A Pearce, ${ }^{1} \mathrm{R}$ Viner, ${ }^{3} \mathrm{~S}$ Morris, ${ }^{4} \mathrm{D}$ Taylor-Robinson, ${ }^{1} \mathrm{H}$ Roberts, ${ }^{1} \mathrm{~S}$ Chigogora. ${ }^{1}$ Population, Policy and Practice Programme, University College London, London, UK; ${ }^{2} \mathrm{MRCI}$ CSO Social and Public Health Sciences Unit, University of Glasgow, Glasgow, UK; ${ }^{3}$ Centre of Applied Health Research, University College London, London, UK; ${ }^{4}$ Department of Public Health and Policy, University of Liverpool, Liverpool, UK

\subsection{6/jech-2019-SSMabstracts.87}

Background About half of lone-parent families in the UK live in relative poverty (income below $60 \%$ of the national median) compared to a quarter of two-parent households, and family hardship is associated with poorer child mental health. There is little evidence on whether reducing income differences between lone- and two-parent households could reduce inequality in child mental health problems (CMHP). Using data from the UK Millennium Cohort Study ( 18000 children born 2000-2002), we investigated whether equalising income between lone- and two-parent households could reduce prevalence and inequality in CMHP. We also simulated achievement of a government target to reduce child poverty to less than $10 \%$ of the population (Child Poverty Act 2010), to assess whether tackling low income could reduce inequality in CMHP related to family structure.

Methods Exposure was family structure (lone- or two-parent household) when the child was $9 \mathrm{~m}$; mediator was equivalised household income per week at 3y; outcome was parent-report CMHP at 5y (Strengths and Difficulties Questionnaire; borderline-abnormal, yes/no). We accounted for attrition to MCS at $5 \mathrm{y}$, and baseline and intermediate confounders. Analyses were carried out in an analytic sample of 11,193.

We estimated the controlled direct effect of family structure on CMHP in logistic marginal structural models, weighted for attrition, and adjusted for confounding and mediation by household income. Prevalence was assessed overall and according to family structure; inequalities were computed using risk ratios (RRs) and differences (RDs) [95\% CIs]. We re-estimated the controlled direct effect after increasing income according to two simulations. In the first, we equalised income between lone- and two-parent households. In the second, we modelled achievement of the child poverty target by increasing income for some lone-and two-parent families, reducing the proportion of households living in relative poverty to less than $10 \%$. Results Prevalence of CMHP was 8.5\% [7.6, 9.5], and children from lone-parent households were more likely to exhibit poorer mental health (RR 1.73; RD 5.70). Equalising income between lone- and two-parent households reduced prevalence $(8.2 \%[7.3,9.0])$, and inequality (RR, 1.37; RD, 2.86) in 
CMHP. Reducing child poverty in both lone- and two-parent households also decreased prevalence in CMHP (7.9\% [7.0, $8.9]$ ), but resulted in smaller reductions in inequality due to family structure (RR 1.63; RD 4.60). Sensitivity analyses showed that associations between exposure, mediator and outcome were comparable across MCS sweeps.

Conclusion Inequalities in CMHP between lone- and twoparent families in the UK are large, but could be reduced by tackling income inequality.

\section{OP85 PREVALENCE OF PTSD AND COMORBIDITY WITH ANXIETY AND DEPRESSION IN A POPULATION-BASED SURVEY OF WOMEN FOLLOWING CHILDBIRTH}

${ }^{1} S$ Harrison*, ${ }^{2} S$ Ayers, ${ }^{1} \mathrm{MA}$ Quigley, ${ }^{1} \mathrm{~F}$ Alderdice. 'National Perinatal Epidemiology Unit, University of Oxford, Oxford, UK; ${ }^{2}$ City, University of London, London, UK

\subsection{6/jech-2019-SSMabstracts.88}

Background Post-traumatic stress disorder (PTSD) can occur after the experience of real or perceived trauma during childbirth or the postpartum period. Estimates of the prevalence and correlates of PTSD following childbirth vary widely in the literature. The aim of this study was to explore: prevalence of PTSD; comorbidity between PTSD, anxiety and depression; and association between PTSD and sociodemographic characteristics in a large sample of women six months after childbirth.

Methods The study was a cross-sectional population-based questionnaire survey of 16,000 women selected at random from birth registrations. The women had given birth in England during October 2017 and were six months postpartum at the time of the survey. The women received a postal questionnaire, which they could complete on paper, by telephone or online. The questionnaire included validated measures of PTSD (Primary Care PTSD Screen (PC-PTSD)), anxiety (Generalised Anxiety Disorder Scale-2) and depression (Edinburgh Postnatal Depression Scale) in addition to general questions covering health and care during pregnancy, labour and birth, and the postnatal period. Prevalence of PTSD, anxiety and depression was estimated with 95\% confidence intervals; association between PTSD and sociodemographic characteristics was explored using logistic regression. Survey weights based on characteristics associated with response were applied to the data.

Results Questionnaires were returned by 4,509 women. The mean age of the women was 32 years ( $\mathrm{sd}=5$ years) and the majority were married (64\%), born in the UK (77\%), and from White British backgrounds (76\%). One in ten women (9.4\%,95\%CI:8.6-10.3) scored above the cut-off on the PCPTSD, reporting at least three of the four symptoms of PTSD (re-experiencing, emotional numbing, avoidance, hyperarousal); $2.5 \%$ of women indicated that their symptoms were specifically related to their childbirth experience (95\% CI:2.1-3.0). The prevalence of anxiety was significantly higher in women with PTSD (score $>3$ ) (45.0,95\%CI:40.150.0) compared to women without PTSD (score $<3$ ) (9.9\%,95\%CI:9.0-10.9). Similarly, the prevalence of depression was significantly higher in women with PTSD (59.3\%,95\%CI:54.3-64.1) compared to women without PTSD (10.7\%,95\%CI:9.8-11.8). Regression analyses indicated that women who were younger, unmarried and born in the UK were more likely to report PTSD.
Conclusion The survey findings indicate a significant proportion of women were experiencing PTSD six months after childbirth. There was high comorbidity between PTSD, anxiety and depression with younger, unmarried, UK-born women being most at risk. Current guidelines recommend screening for anxiety and depression during the postnatal period; a brief screen for PTSD should be included to ensure women with symptoms are identified and offered focused interventions.

\section{OP86 PREVALENCE OF DEPRESSION AMONG PEOPLE WITH DIABETES; A COMPARATIVE ANALYSIS OF ADULTS ACROSS THREE HEALTH SYSTEMS USING NATIONALLY REPRESENTATIVE DATA}

${ }^{1} \mathrm{~N}$ McGrath*, 'S McHugh, ${ }^{2} \mathrm{E}$ Toomey, ${ }^{1}$ PM Kearney. 'School of Public Health, University College Cork, Cork, Ireland; ${ }^{2}$ School of Psychology, National University of Ireland, Galway, Galway, Ireland

\subsection{6/jech-2019-SSMabstracts.89}

Background Cross country comparison facilitates examination of health-system and country-level similarities and differences on disease burden. Diabetes is a leading global health issue with outcomes influenced by psychological comorbidity. Diabetes is consistently associated with depression in different populations but any association is influenced by variations in prevalence, study design and measure used. We compared depressive symptom prevalence in older adults with and without diabetes across three health systems using a validated measure to examine the causal association between diabetes and depression.

Methods We used data of adults aged 50 years and older, from three nationally representative ageing datasets; The Irish Longitudinal Study on Ageing (TILDA), the English Longitudinal Study on Ageing (ELSA) and the Health and Retirement Study (HRS). Data were collected during 2009-2011 (TILDA), and 2010 (ELSA, HRS). Variables; country, gender, age, education level, marital status and smoking status, were selected a priori using literature and a directed acyclic graph. Variables were self-report, with the exception of 'country', which was linked to the original dataset. The 20-item (cut-off-score $>16$; TILDA) and 8-item CESD (cut-off-score $>3$;ELSA and HRS) were used to categorise depression. Prevalence was presented as a percentage with corresponding 95\% confidence intervals and group-specific differences presented using Pearson's chisquare test for categorical data and Student's t-test for continuous data. Binomial logistic regression examined the odds of depression by diabetes status, adjusting for pre-selected variables. Analyses of country-specific factors related to depression is ongoing. Data were analysed using Stata v15.

Results Diabetes prevalence varied across the countries (Ireland; 8\%[(95\%CI:7.5-8.6), England; 11\%(95\%CI:10.6-12.0), USA; 22\%[(95\%CI:21.7-22.8); $<<0.001])$ and was consistently higher among males than females (Ireland; 10\%(95\%CI:8.9$10.8)$ vs. $6 \%(95 \% \mathrm{CI}: 5.3-6.7)$, England; 13\%(95\%CI:12.4 $14.5)$ vs. $10 \%(95 \% \mathrm{CI}: 8.7-10.3)$, USA; 24\%(95\%CI:22.9-24.7) vs. $21 \%[(95 \% \mathrm{CI}: 20.3-21.8) ; \mathrm{p}<0.001])$. Depression prevalence was significantly higher among people with diabetes in all countries (Ireland; 12\%(95\%CI:9.8-15.1) vs. $10 \%[(95 \%$ CI:9.0-10.4), $\mathrm{p}=0.035]$, England; 34\%(95\%CI:31.0-37.0) vs. 22\%[(95\%CI:20.8-22.6):p<0.001], USA; 20\%(95\%CI:19.0$21.4)$ vs. $14 \%[(95 \% \mathrm{CI}: 13.6-14.7) ; \mathrm{p}<0.001]$, in females (Ireland; $19 \%(95 \% \mathrm{CI}: 14.6-23.9) \quad$ vs. $12 \%[(95 \% \mathrm{CI}: 11.0-12.9)$; $\mathrm{p}=0.001]$, England; 39\%(95\%CI:34.2-43.4) vs. $25 \%[(95 \%$ 